\title{
Modified QuEChERS Based on Mixed Adsorbent Cleanup of Mycotoxins in Corn
}

\author{
Liyuan Zhang $\mathbb{D}^{1,2,3}$ Anna Dai, ${ }^{1}$ Runzhong Yu $\mathbb{D}^{2,4}$ and Lili Qian ${ }^{1,3}$ \\ ${ }^{1}$ College of Food Science, Heilongjiang Bayi Agricultural University, 5 Xinfeng Road, Daqing 163319, China \\ ${ }^{2}$ Heilongjiang Province Cultivating Collaborative Innovation Center for the Beidahuang \\ Modern Agricultural Industry Technology, Daqing 163319, China \\ ${ }^{3}$ Key Laboratory of Agro-Products Processing and Quality Safety of Heilongjiang Province, Daqing 163319, China \\ ${ }^{4}$ College of Electrical and Information, Heilongjiang Bayi Agricultural University, 5 Xinfeng Road, Daqing 163319, China
}

Correspondence should be addressed to Liyuan Zhang; zly1981_2005@163.com and Runzhong Yu; yrz0459@163.com

Received 4 March 2020; Revised 19 April 2020; Accepted 21 April 2020; Published 20 May 2020

Academic Editor: Pedro M. Mancini

Copyright ( 2020 Liyuan Zhang et al. This is an open access article distributed under the Creative Commons Attribution License, which permits unrestricted use, distribution, and reproduction in any medium, provided the original work is properly cited.

In order to analyze the mycotoxins in corn, the modified QuEChERS high-performance liquid chromatography was applied to extract, clean up, and detect mycotoxins in a nonpolar system. The impurities such as fat and protein were removed from the corn sample, and the impurities in the sample solution have almost no effect on extraction efficiency. The proposed method leads to a greater choice of mycotoxin-extraction solvents in high-fat-solid samples. An appropriate extraction solvent was selected, the pretreatment conditions were optimized, and the extraction and cleanup of mycotoxins in high-fat solids were enhanced. By changing the experiment parameters, this method can be further used for the extraction and analysis of mycotoxins in complex samples (nonfat, low-fat, or high-fat). This method achieves good linearity in the range of $2.5-1000 \mu \mathrm{g} / \mathrm{kg}$ with the correlation coefficients for all analytes in the range of 0.9975 to 0.9989 . The acceptable standard deviations for intraday and interday precision were $1.8-4.3 \%$ and $3.2-5.2 \%$, respectively, with recoveries from 89.7 to $105.9 \%$.

\section{Introduction}

In 1960, about 100,000 turkeys died from acute aflatoxin poisoning in the United Kingdom. After aflatoxin was confirmed to be seriously carcinogenic in the following year, mycotoxins began attracting widespread attention $[1,2]$. Mycotoxins are a series of toxic secondary metabolites produced by some molds (mainly aspergillus, penicillium, and fusarium) after they mature [3-5]. They can be divided into field toxins and storage toxins, depending on where they grow $[6,7]$. The identification and classification of molds have traditionally been based on morphological and cultural characteristics, but the morphological characteristics of molds are complex, and morphological studies of them are often questioned on the basis of lack of standards and excessive subjectivity. The common mycotoxin is aflatoxins (AFs, including $\mathrm{AFB}_{1}, \mathrm{AFB}_{2}, \mathrm{AFG}_{1}$, and $\mathrm{AFG}_{2}$ ), zearalenone (ZEN), deoxynivalenol (DON), sterigmatocystin (ST), citreoviridin (CIT), ochratoxin A (OTA), and so on [8]. Mycotoxins seriously endanger the health of livestock and poultry $[9,10]$ and, thereby, indirectly endanger human health, leading to severe economic losses to the companies producing them $[11,12]$. The $\mathrm{WHO}$ and the FAO have identified mycotoxins as the main cause of foodborne illness. Globally, mycotoxins contaminate 25 percent of food according to the FAO. Therefore, rapid and effective detection of mycotoxins can prevent their occurrence and improve the quality and safety of animal products.

Various regions have established maximum residue limit (MRL) of mycotoxin standards to regulate it. China's national standards stipulate that the MRLs of $\mathrm{AFB}_{1}, \mathrm{DON}$, ZEN, and OTA in corn are $20,1000,60$, and $5 \mu \mathrm{g} / \mathrm{kg}$, respectively (GB 2761-2017). In the European Union, the MRLs of $\mathrm{AFB}_{1}, \mathrm{DON}, \mathrm{ZEN}$, and OTA in corn are 2.0, 1750, 350 , and $5 \mu \mathrm{g} / \mathrm{kg}$, respectively. In the U.S., the MRLs of $\mathrm{AFB}_{1}$, DON, ZEN, and OTA in corn are 5.0, 4000, 350, and $5 \mu \mathrm{g} / \mathrm{kg}$, 
respectively. However, the MRLs of $\mathrm{AFB}_{2}, \mathrm{ST}$, and CIT in corn are not specified. And the classification of standard is still not specific enough to effectively distinguish the limit value of raw grain and finished grain [13-15]. Therefore, it is necessary to continuously improve the relevant analysis methods, which will help to come up with a more reasonable limit standard to ensure the corn food security.

At present, commonly used pretreatment methods are liquid-phase microextraction [16-18], liquid-liquid microextraction [19], dispersive liquid-liquid microextraction [20-22], QuEChERS [23-25], SPE [26], and DSPE [27]. Most of these methods are used for the extraction of a single mycotoxin. There are few reports on the analysis of several mycotoxins. Compared with other methods, QuEChERS is more and more widely used due to its advantages of quick, easy, cheap, effective, rugged, and safe. The association of official analytical chemists (AOAC) and European committee for standardization (EN) have released the method standard based on this technology (AOAC 2007.01 and EN $15662: 2008)[28,29]$. In order to expand the use of the QuEChERS method, this work improved it.

In this work, the modified QuEChERS procedure was developed to extract, separate, and clean up mycotoxins in corn samples. A mixture of ethyl acetate and n-hexane was used as an extraction solvent. The extraction and cleanup can be completed in one step, and analysis speed can be greatly improved.

\section{Materials and Methods}

2.1. Sample. Three kinds of corn in the grain storehouse were randomly selected and simulated in a container transportation environment. The samples were divided into sample 1, sample 2, and sample 3, and the samples were crushed to ensure that $75 \%$ of the corn samples passed through an 80 -mesh sieve. All samples were sealed and stored at $-20^{\circ} \mathrm{C}$ for testing.

2.2. Reagents and Equipment. The main apparatus are listed in Supplementary Table 1. Acetonitrile and methanol (chromatographical pure) were purchased from ThermoFisher, USA. The formic acid and ammonium formate were purchased from Sigma-Aldrich, USA. All other reagents (analytical grade) were purchased from the Beijing Chemical Factory (Beijing, China). The water used in the experiment was Milli-Q ultrapure water.

Mycotoxin standards, including aflatoxin $\mathrm{B}_{1}\left(\mathrm{AFB}_{1}\right)$, aflatoxin $\mathrm{B}_{2}\left(\mathrm{AFB}_{2}\right)$, sterigmatocystin (ST), and citreoviridin (CIT), were prepared in acetonitrile at a concentration of $100 \mu \mathrm{g} / \mathrm{mL}$ as a standard stock solution. All standard stock solutions were stored in a refrigerator at $4^{\circ} \mathrm{C}$. A standard working solution $(10 \mu \mathrm{g} / \mathrm{mL})$ was prepared by diluting the standard stock solution with acetonitrile weekly.

As mycotoxins are extremely toxic, endangering health and polluting the environment, it is necessary to take good care during the experiment to avoid contact between mycotoxins and the skin. The containers, consoles, and items that have been exposed to mycotoxins must be disinfected with a 5\% sodium hypochlorite-acetone solution. The containers should be immersed in a closed disinfectant for at least 30 minutes and then washed with water.

2.3. Chromatographic Conditions. The analytical column was Eclipse XDB-C18 column $(150 \mathrm{~mm} \times 4.6 \mathrm{~mm}$ i.d., $3.5 \mu \mathrm{m}$, Agilent, USA), the column temperature was $35^{\circ} \mathrm{C}$, mobile phase A had $0.1 \%$ formic acid aqueous solution, mobile phase B had methanol, gradient elution procedure was carried out: $0.0 \mathrm{~min} \longrightarrow 2.0 \mathrm{~min}, 5 \% \quad \mathrm{~B} ; 2.0 \mathrm{~min}$ $\longrightarrow 10.0 \mathrm{~min}, 5 \% \mathrm{~B} \longrightarrow 50 \% \mathrm{~B} ; 10.0 \mathrm{~min} \longrightarrow 12.0 \mathrm{~min}, 50 \%$ $\mathrm{B} \longrightarrow 60 \% \mathrm{~B}$; and $12.0 \mathrm{~min} \longrightarrow 25.0 \mathrm{~min}, 60 \% \mathrm{~B} \longrightarrow 90 \% \mathrm{~B}$, the flow rate was $0.5 \mathrm{~mL} / \mathrm{min}$, and injection volume was $10 \mu \mathrm{L}$. The DAD detection was carried out at a wavelength of $225 \mathrm{~nm}$. The reference wavelength and bandwidth were 360 and $4 \mathrm{~nm}$, respectively.

2.4. Modified QuEChERS Procedure. The $5.000 \mathrm{~g}$ (to within $0.001 \mathrm{~g}$ ) corn-powder samples were accurately weighed and added into a $50 \mathrm{~mL}$ centrifuge tube with a lid, and $16 \mathrm{~mL}$ $0.5 \%$ (volume concentration) formic-acid aqueous solution was added. Then, the mixed sample solution was homogenized in a high-speed homogenizer. After $30 \mathrm{~s}$, the tube was vigorously shaken for $5 \mathrm{~min}$ and the $6 \mathrm{~mL}$ extraction solvent (ethyl acetate: $\mathrm{n}$-hexane $=1: 1$ ) and $0.3 \mathrm{~g} \mathrm{NaCl}$ were added into the tube. The ultrasonic extraction was carried out for $5 \mathrm{~min}$, the mixed solution was centrifuged at $0^{\circ} \mathrm{C}$ and $15,000 \mathrm{rpm}$ for $5 \mathrm{~min}$, and the supernatant was transferred into another centrifuge tube containing $80 \mathrm{mg}$ of adsorbent ( $40 \mathrm{mg}$ of diatomaceous earth $+40 \mathrm{mg}$ PSA); after shaking, the mixture was centrifuged at $15,000 \mathrm{rpm}$ for $5 \mathrm{~min}$, then the supernatant was transferred to a glass flask and evaporated to dryness at $35^{\circ} \mathrm{C}$ in a vacuum rotary evaporator, and the residue was redissolved with $100 \mu \mathrm{l}$ of solvent (acetonitrile). Finally, the analytical solution was filtered with a PTFE filter and analyzed.

\subsection{Method Evaluation}

2.5.1. Linear Range. A standard curve was prepared from the peak area versus the analyte concentration. The linearity of the data was evaluated using a linear correlation coefficient.

2.5.2. Precision and Recovery. For the same sample, analyze it 5 times a day to find the intraday precision. For the same sample, analyze it once a day for five days to get the interday precision. The intraday and interday precisions are expressed as relative standard deviations (RSDs). Then, the average extraction recoveries were obtained.

2.5.3. Limit of Detection and Limit of Quantitation. The detection limit (LOD) and the lower limit of quantification (LOQ) are the lowest concentration values when the signalto-noise ratio is 3 and 10 times, respectively. 
Table 1: Analytical performance.

\begin{tabular}{lccccc}
\hline Mycotoxin & Regression equation & Correlation coefficient & Linear range $(\mu \mathrm{g} / \mathrm{kg})$ & $\mathrm{LOD}(\mu \mathrm{g} / \mathrm{kg})$ & $\mathrm{LOQ}(\mu \mathrm{g} / \mathrm{kg})$ \\
\hline $\mathrm{AFB}_{1}$ & $A=138.54 c+19.04$ & 0.9981 & $2.5-1000$ & 3.5 & 10.4 \\
$\mathrm{AFB}_{2}$ & $A=102.56 c+16.03$ & 0.9989 & $2.5-1000$ & 4.8 & 16.3 \\
$\mathrm{ST}$ & $A=54.82 c+5.63$ & 0.9975 & $2.5-1000$ & 7.2 & 35.1 \\
$\mathrm{CIT}$ & $A=39.53 c+7.21$ & 0.9987 & $2.5-1000$ & 6.3 & 20.6 \\
\hline
\end{tabular}

TABLE 2: Analytical results of samples.

\begin{tabular}{|c|c|c|c|c|c|}
\hline Sample & Mycotoxin & Added level $(\mu \mathrm{g} / \mathrm{kg})$ & Recovery (\%) & Intra-RSD (\%) & Inter-RSD (\%) \\
\hline \multirow{8}{*}{1} & \multirow{2}{*}{ AFB1 } & 10.0 & 104.2 & 1.8 & 3.4 \\
\hline & & 100.0 & 103.1 & 1.7 & 3.6 \\
\hline & \multirow{2}{*}{ AFB2 } & 10.0 & 101.8 & 2.0 & 4.1 \\
\hline & & 100.0 & 102.9 & 1.8 & 3.2 \\
\hline & \multirow{2}{*}{ ST } & 10.0 & 95.0 & 2.2 & 4.5 \\
\hline & & 100.0 & 98.3 & 1.9 & 3.6 \\
\hline & \multirow{2}{*}{ CIT } & 10.0 & 89.7 & 1.9 & 3.9 \\
\hline & & 100.0 & 92.1 & 2.4 & 5.2 \\
\hline \multirow{8}{*}{2} & \multirow{2}{*}{ AFB1 } & 10.0 & 102.6 & 1.9 & 3.7 \\
\hline & & 100.0 & 103.5 & 1.8 & 3.6 \\
\hline & \multirow{2}{*}{ AFB2 } & 10.0 & 100.6 & 2.0 & 4.3 \\
\hline & & 100.0 & 103.0 & 2.1 & 4.2 \\
\hline & \multirow{2}{*}{$\mathrm{ST}$} & 10.0 & 93.4 & 2.3 & 4.2 \\
\hline & & 100.0 & 95.5 & 2.1 & 4.8 \\
\hline & \multirow{2}{*}{ CIT } & 10.0 & 96.5 & 2.4 & 5.0 \\
\hline & & 100.0 & 94.8 & 2.5 & 5.1 \\
\hline \multirow{8}{*}{3} & \multirow{2}{*}{ AFB1 } & 10.0 & 102.9 & 2.0 & 4.7 \\
\hline & & 100.0 & 104.5 & 2.2 & 4.3 \\
\hline & \multirow{2}{*}{ AFB2 } & 10.0 & 99.8 & 2.1 & 4.1 \\
\hline & & 100.0 & 102.7 & 1.9 & 3.6 \\
\hline & \multirow{2}{*}{ ST } & 10.0 & 97.4 & 1.7 & 3.2 \\
\hline & & 100.0 & 95.3 & 2.1 & 3.6 \\
\hline & \multirow{2}{*}{ CIT } & 10.0 & 90.2 & 1.9 & 4.1 \\
\hline & & 100.0 & 91.4 & 1.8 & 3.2 \\
\hline
\end{tabular}

\section{Results and Discussion}

\subsection{Optimization of Extraction Conditions}

3.1.1. Effects of Type and Volume of Extraction Solvent. The experiments examined the effects of acetonitrile (ACN), acetone (AO), ethyl acetate (EA), n-hexane (HA), and ethyl acetate/ n-hexane (EA/HA, V: V=1:1) on mycotoxin extraction. As shown in Supplementary Figure 1, due to the strong polarity of acetonitrile, it cannot effectively penetrate high-fat samples and is not conducive to the extraction of analytes. N-hexane is nonpolar, but although it can effectively penetrate the sample, its ability to dissolve the more polar mycotoxin is limited, resulting in lower recovery of target analytes. The ethyl acetate is cheap, has low toxicity, evaporates easily, and has medium polarity. Ethyl acetate has a more suitable polarity and is more conducive to the extraction of target analytes with similar polarity. Compared with other extraction solvents, the interference peaks of the chromatogram obtained by ethyl acetate/n-hexane extraction were less and the baseline noise was lower. After extraction of ethyl acetate/n-hexane, the target analytes are adsorbed and the fat remains in the solution, thereby eliminating its effects on the extraction of the analytes.
Therefore, ethyl acetate and n-hexane (EA/HA, V: V=1:1) were selected as the extraction solvents in this experiment.

The effect of volume of the extraction solvent in the range of 2.00 to $8.00 \mathrm{~mL}$ on extraction of the analytes was also investigated. As the volume of the extraction solvent increases, the recoveries of the analytes increase first and then remain constant. At $6.00 \mathrm{~mL}$, maximum recoveries of analytes are achieved, and therefore $6.00 \mathrm{~mL}$ of ethyl acetate and $\mathrm{n}$-hexane $(1: 1)$ was used in this experiment.

3.1.2. Effects of Ultrasonic Extraction Time. Ultrasonic extraction is an equilibrium extraction, and the optimal extraction efficiency is established by equilibrium. The effects of the ultrasonic extraction time on recoveries of mycotoxins were evaluated by performing a single factor experiment taking 2-6 min. The results are shown in Supplementary Figure 2. When the extraction time increased from 2 to $5 \mathrm{~min}$, the peak quantity of analytes increased significantly, but the recoveries did not change as the extraction time continued to increase. This may be because the surface area of extraction solvent and aqueous solution is large, the target analytes transfer quickly between the two phases, the extraction equilibrium can be reached in a very short time, and 


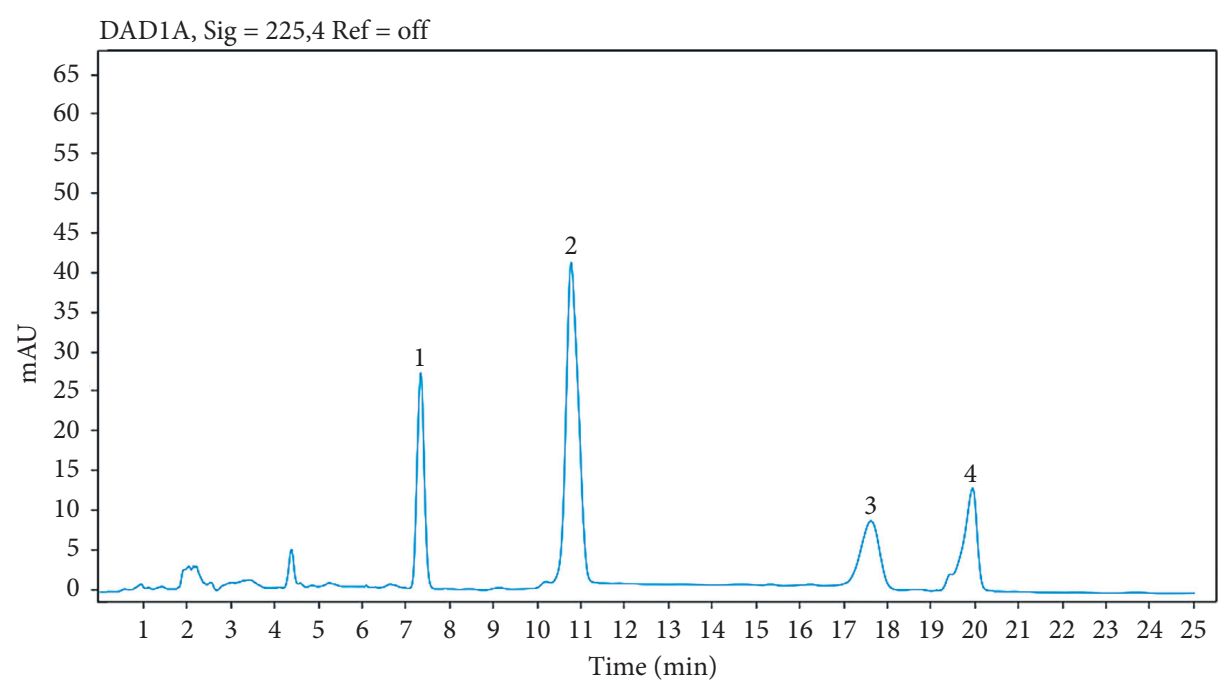

(a)

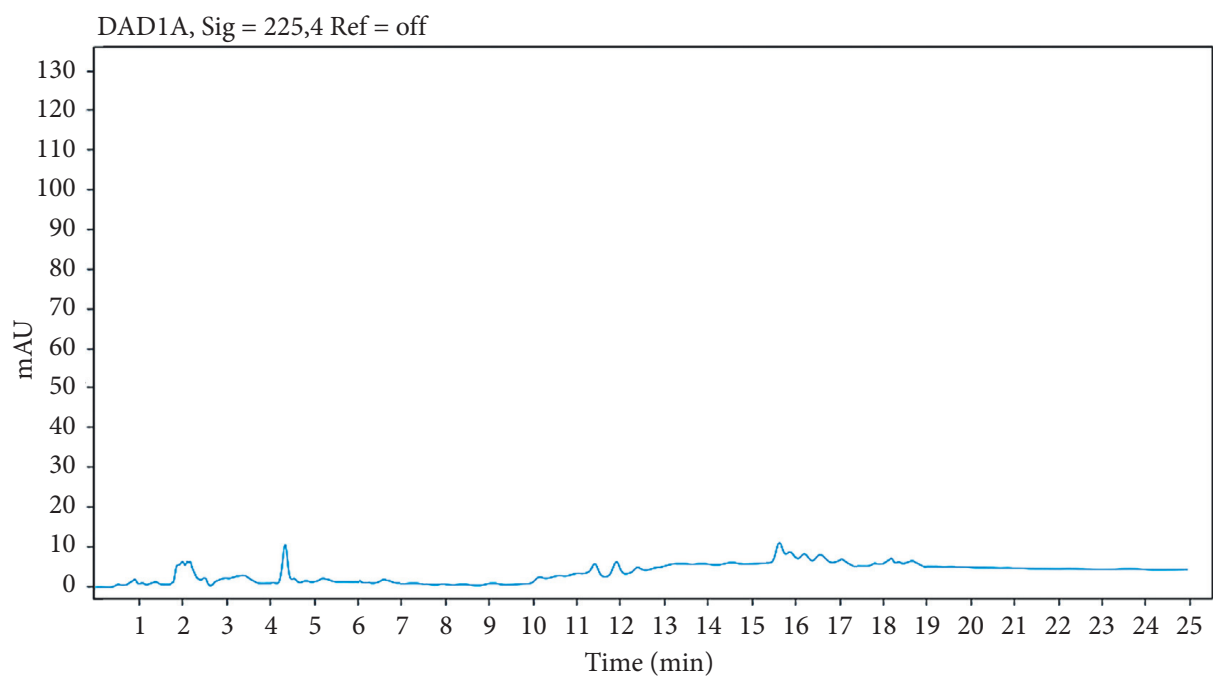

(b)

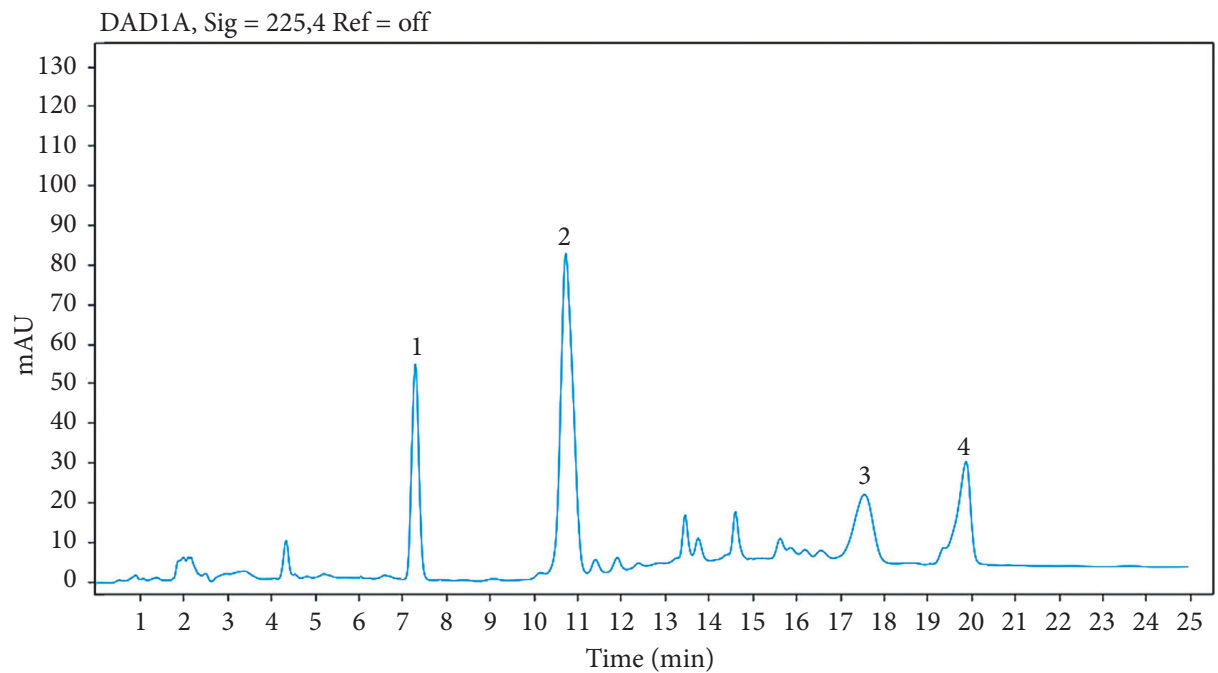

(c)

Figure 1: HPLC chromatograms. (a) Standard solution, (b) blank sample, and (c) spiked sample (10 $\mu \mathrm{g} / \mathrm{kg}) .1$ AFB1, 2 AFB2, 3 ST, and 4 CIT. 
TABLE 3: Comparison of the proposed method with other methods.

\begin{tabular}{|c|c|c|c|c|c|c|c|c|c|}
\hline Matrix & Method* & $\begin{array}{c}\text { Sample } \\
\text { amount (g) }\end{array}$ & $\begin{array}{c}\text { Organic } \\
\text { solvent } \\
(\mathrm{mL})\end{array}$ & $\begin{array}{l}\text { The linear } \\
\text { range }\end{array}$ & $\begin{array}{l}\text { Extraction } \\
\text { time (min) }\end{array}$ & $\begin{array}{l}\text { Spiked } \\
\text { level }\end{array}$ & $\begin{array}{l}\text { Recovery } \\
(\%)\end{array}$ & RSD (\%) & Reference \\
\hline Corn & $\begin{array}{c}\text { Modified } \\
\text { QuEChERS- } \\
\text { HPLC }\end{array}$ & 5.0 & 6.1 & $\begin{array}{c}2.5 \sim 1000 \\
(\mu \mathrm{g} / \mathrm{kg})\end{array}$ & 2 & $\begin{array}{l}10,100 \\
(\mu \mathrm{g} / \mathrm{kg})\end{array}$ & $89.7 \sim 105.9$ & $1.8 \sim 4.3$ & This work \\
\hline Juice & $\begin{array}{c}\text { AS-DLLME-LC- } \\
\text { MS }\end{array}$ & 5.0 & 2.6 & $\begin{array}{l}1 \sim 500 \\
(\mu \mathrm{g} / \mathrm{L})\end{array}$ & 20 & $\begin{array}{l}0.2,1,10 \\
(\mu \mathrm{g} / \mathrm{kg})\end{array}$ & $75.6 \sim 110.0$ & $1.4 \sim 15.5$ & [22] \\
\hline Feed & $\begin{array}{l}\text { QuEChERS- } \\
\text { UHPLC-MS/MS }\end{array}$ & 2.0 & 10.5 & $\begin{array}{l}1 \sim 500 \\
(\mu \mathrm{g} / \mathrm{L})\end{array}$ & 21 & $\begin{array}{c}5,20,100 \\
(\mu \mathrm{g} / \mathrm{kg})\end{array}$ & $89.3 \sim 112.6$ & $0.9 \sim 6.1$ & [23] \\
\hline Cereal & $\begin{array}{c}\text { QuEChERS- } \\
\text { HPLC }\end{array}$ & 2.0 & 10.1 & $\begin{array}{c}0.66 \sim 150 \\
(\mu \mathrm{g} / \mathrm{kg})\end{array}$ & 5 & $\begin{array}{l}2,20 \\
(\mu \mathrm{g} / \mathrm{kg})\end{array}$ & $60 \sim 98$ & 7.2 12.1 & [24] \\
\hline Notoginseng & $\begin{array}{l}\text { QuEChERS-- } \\
\text { UHPLC-MS/MS }\end{array}$ & 2.0 & 11 & $\begin{array}{c}0.25 \sim 125 \\
(\mu \mathrm{g} / \mathrm{L})\end{array}$ & 102 & $20(\mu \mathrm{g} / \mathrm{L})$ & $80.1 \sim 116.2$ & $0.2 \sim 5.7$ & [25] \\
\hline Oil & SPE-HPLC & 5.0 & 26 & $\begin{array}{c}0.5 \sim 50 \\
(\mu \mathrm{g} / \mathrm{L})\end{array}$ & 60 & $\begin{array}{l}0.5,1,2 \\
(\mu \mathrm{g} / \mathrm{kg})\end{array}$ & $81.4 \sim 105.3$ & $1.3 \sim 8.6$ & [26] \\
\hline Oil & DSPE-HPLC & 2.0 & 10.5 & $\begin{array}{c}50 \sim 40000 \\
(\mu \mathrm{g} / \mathrm{L})\end{array}$ & 32 & $\begin{array}{c}0.2 \sim 8 \\
(\mu \mathrm{g} / \mathrm{kg})\end{array}$ & $60 \sim 120$ & $4.16 \sim 25.2$ & [27] \\
\hline
\end{tabular}

the extraction efficiency will not be affected by the increase of extraction time. Therefore, the time we selected was $5 \mathrm{~min}$.

3.1.3. Effects of Type of Cleanup Adsorbent. The effects of different cleanup adsorbents, including C18, diatomite, PSA, neutral alumina $\mathrm{Al}_{2} \mathrm{O}_{3}$, and diatomaceous earth + PSA $(1: 1)$, on recoveries of target analytes were surveyed. As shown in Supplementary Figure 3, the recoveries of mycotoxins are the highest when the mixed adsorbent of diatomaceous earth and PSA was used as clean-up adsorbent. There are two possible reasons. On one hand, mycotoxins are polar molecular substances, which are a molecule with strong anode polarity. When selecting a cleanup adsorbent, it is necessary to have a molecular structure with polarity and the capacity for ion exchange. Different cleanup adsorbents have different capacities for absorbing mycotoxins. The zeolite and bentonite are unipolar and swell in water. They can adsorb both aflatoxins and water-soluble nutrients. On the other hand, the mixed cleanup adsorbent of diatomaceous earth and PSA, which is bipolar, has the best cation exchange balance, does not swell in water, has a strong ability to adsorb toxins, and has a wide range. It can adsorb not only aflatoxin, but also vomiting toxin, zearalenone, and T-2 toxin. However, it does not absorb nutrients. Therefore, when diatomaceous earth and PSA were mixed, the four mycotoxins had the highest recoveries. Hence, the diatomaceous earth + PSA $(1: 1)$ was selected as a cleanup adsorbent in further work.

\subsubsection{Effects of Amount of Mixed Cleanup Adsorbent.} The experiment also examined the effect on the recoveries when the amount of the mixed adsorbent was changed from $40 \mathrm{mg}$ to $250 \mathrm{mg}$. The results (Supplementary Figure 4) showed that the amount of adsorbent was too small to be sufficiently purified, and the recoveries of the mycotoxin analytes increased with the increase in the amount of the mixed adsorbent. When the mixed adsorbent amounted to $80 \mathrm{mg}$, the recoveries reached its highest point. This is because when the amount of adsorbent is greater than $80 \mathrm{mg}$, the cleanup adsorbent will have a stronger adsorption on the mycotoxin, which is not easy to be eluted, and on the contrary, the recoveries will be reduced. So, $80 \mathrm{mg}$ was the amount of the cleanup adsorbent in further work.

\subsection{Method Evaluation}

3.2.1. Linear. A series of standard solution of mycotoxins was prepared, respectively. The above standard solution of $20 \mu \mathrm{L}$ was injected into the high-performance liquid chromatograph. The standard curves were drawn with the concentration of analytes in the standard solution as the abscissa, and the peak areas of chromatogram were as the ordinate. The recoveries of the analytes were calculated according to the standard curve. All concentrations were tested in quintuplicate. The formula for calculating the recovery is

recovery $=\frac{\text { change in the amount of measured analyte }}{\text { amount of analyte spiked into the sample }} \times 100 \%$.

The linear regression equations and correlation coefficients are listed in Table 1. This method achieves good linearity with correlation coefficients for all analytes in the range of 0.9975 to 0.9989 .

3.2.2. Precision and Recovery. The precision of the method was assessed by measuring intraday and interday precision. Intraday precision is measured of corn samples with a concentration of 10 and $100 \mu \mathrm{g} / \mathrm{kg}$ five times in one day. Interday precision is measured of corn samples with a concentration of 10 and $100 \mu \mathrm{g} / \mathrm{kg}$ once a day for five consecutive days. Table 2 lists the relative standard deviations and recoveries. The results showed that the acceptable standard deviations for intraday and interday precision were $1.8-4.3 \%$ and $3.2-5.2 \%$, respectively, with recoveries from 89.7 to $105.9 \%$. 
3.2.3. Limit of Detection and Limit of Quantitation. The limits of detection (LOD) and the limit of quantitation (LOQ) are the concentration values of the analytes when the signal of the measured object is 3 times and 10 times of the noise signal, respectively. As shown in Table 1, the limits of detection $(\mathrm{LOD}, \mathrm{S} / \mathrm{N}$ ratio $=3$ ) for all analytes range from 3.5 to $7.2 \mu \mathrm{g} / \mathrm{kg}$. The limit of quantitation (LOQ, S/N ratio $=10$ ) for all analytes is below $10 \mu \mathrm{g} / \mathrm{kg}$, which is below the MRL stipulated by the European Union.

3.2.4. Sample Analysis. The analysis of the actual sample can get good recoveries (89.7-105.9\%) and precision ( $\leq 5.2 \%)$. The chromatograms are shown in Figures 1(a)-1(c). Table 2 shows the actual results. The $\mathrm{AFB}_{1}, \mathrm{AFB}_{2}, \mathrm{ST}$, and CIT in three kinds of corn were undetectable.

3.3. Comparison of the Proposed Method with Other Methods. The proposed method was compared with [22-27]. As shown in Table 3, the proposed method has four advantages. Firstly, the proposed method uses simpler instruments and equipment, such as centrifuge and oscillator. Secondly, the method has lower technical difficulty and simpler pretreatment steps. Thirdly, it takes less time. The whole extraction process does not exceed 7 min. Finally, the simultaneous extraction and cleanup of four mycotoxins can be realized by designing the proportion of extraction solution, the types of salt, and purifying agents.

\section{Conclusion}

According to the characteristics of QuEChERS, a modified QuEChERS method for extraction and cleanup of mycotoxins in corn was established and developed. The effects of type and volume of extraction solvents, ultrasonic extraction time, and cleanup adsorbent on recovery of mycotoxins during the pretreatment of mycotoxins by the modified QuEChERS method were discussed. Compared with other methods, this method is simpler to operate, the detection time is shortened to less than 7 minutes, and the amount of organic reagent is less, which is suitable for the detection of large amount of samples. The proposed method can effectively overcome the errors caused by sample pretreatment and matrix effect. It expands the choice of mycotoxin extraction solvents in high-fat-solid samples. Choosing a suitable solvent and optimizing the mixed adsorbent was a promising approach to improving the efficiency of extraction and purification of the mycotoxin in the high-fat-solid samples. By changing the experiment parameters, the method could be further used for extraction and analysis of mycotoxins in complex samples.

\section{Data Availability}

The data used to support the findings of this study are available from the corresponding author upon request.

\section{Conflicts of Interest}

The authors declare that they have no known competing financial interest or personal relationships that could have appeared to influence the work reported in this paper.

\section{Acknowledgments}

This work was supported by the National Key Research and Development Program of China (grant number 2018YFD0401403), Heilongjiang Bayi Agricultural University for San Heng San Zong (grant number ZRCQC201906), and Science and Technology Research Project of Heilongjiang Agricultural Reclamation Administration (grant number HKKY190407).

\section{Supplementary Materials}

Supplementary Table 1: the list of apparatus. Supplementary Figure 1: effect of the type of extract solvents on the recoveries of the analytes. Supplementary Figure 2: effect of ultrasonic extraction time on the recoveries of the analytes. Supplementary Figure 3: effect of the type of adsorbents on recoveries of analytes. Supplementary Figure 4: effect of the amount of adsorbents on the recoveries of the analytes. (Supplementary Materials)

\section{References}

[1] Y. Zheng, Analysis of Aflatoxin and its Degradation Products in Food Based on LC-MS, Nanchang University, Nanchang, China, 2011.

[2] Y. J. Xv and R. J. Fang, "Mycotoxins and their mechanisms," Feed Industry, vol. 4, pp. 52-57, 2008.

[3] Y. L. Chen, "Research progress in detection technology of mycotoxins in grain," China Food Safety Magazine, vol. 135, no. 12 , p. $48,2016$.

[4] D. Mao, Study on the Detection Methods of Mycotoxins in 4 Kinds of Fruit Seeds, Shanghai University of Traditional Chinese Medicine, Shanghai, China, 2015.

[5] R. Z. Wang, X. T. Zhao, Q. B. Lin, and H. Song, "Research progress in analysis of mycotoxins," Chinese Journal of Health Laboratory Technology, vol. 11, pp. 420-425, 2010.

[6] J. J. Luo, B. Zhang, and Y. G. Chen, "Recent research progress of mycotoxins," Hunan Feed, vol. 6, pp. 28-30, 2010.

[7] Y. Z. Feng, S. Wei, Z. S. Wang et al., "Research progress of mycotoxins," Feed Industry, vol. 35, no. 4, pp. 58-62, 2014.

[8] Z. W. Xv and J. Wan, "Research progress of feed mycotoxin adsorbent," Chinese Journal Of Animal Nutrition, vol. 12, no. 5, pp. 1-8, 2019.

[9] H. T. Shi, Z. J. Cao, J. Li, C. H. Guo, Y. Wang, and S. L. Li, "Status and research progress of feed mycotoxins in China," Journal of Southwest Minzu University (Natural Science Edition), vol. 45, no. 4, pp. 354-366, 2019.

[10] C. F. Xiao and M. F. Zhu, "Research progress of mycotoxins in feed," Shanghai Journal of Animal Husbandry and Veterinary Medicine, vol. 4, p. 22, 2014.

[11] G. Y. Fan, In Vitro Evaluation of Corn Major Mycotoxins and Development of Adsorbents, Shandong Agricultural University, Tai'an, China, 2015. 
[12] J. Xv, H. X. Zhang, and T. T. Qu, "Harm of mold and mycotoxins to corn and countermeasures and suggestions," Journal of Heilongjiang Grain, vol. 9, pp. 43-44, 2017.

[13] GB 2761-2017, Limits of Mycotoxins in Food National Food Safety Standards, China standard press, Beijing, China, 2017.

[14] Commission Regulation (EC) No1881/2006, Setting Maximum Levels for Certain Contaminants in FoodstuffsOfficial Journal of the European Union, the Commission of the European Communities, Europe, 2006.

[15] U S Food and Drug Administration, "Guidance for indus-try: fumonisin levels in human foods and animal feeds; Availability [J/OL]," Federal Register, vol. 66, pp. 56-68, 2001.

[16] M. R. A. Mogaddam, A. Mohebbi, A. Pazhohan, F. Khodadadeian, and M. A. Farajzadeh, "Headspace mode of liquid phase microextraction: a review," TrAC Trends in Analytical Chemistry, vol. 110, pp. 8-14, 2019.

[17] M. A. Farajzadeh, A. Shahedi Hojghan, and M. R. Afshar Mogaddam, "Development of a new temperature-controlled liquid phase microextraction using deep eutectic solvent for extraction and preconcentration of diazinon, metalaxyl, bromopropylate, oxadiazon, and fenazaquin pesticides from fruit juice and vegetable samples followed by gas chromatography-flame ionization detection," Journal of Food Composition and Analysis, vol. 66, no. 66, pp. 90-97, 2018.

[18] M. A. Farajzadeh, M. Bamorowat, and M. R. A. Mogaddam, "Ringer tablet-based ionic liquid phase microextraction: application in extraction and preconcentration of neonicotinoid insecticides from fruit juice and vegetable samples," Talanta, vol. 160, pp. 211-216, 2016.

[19] M. Torbati, A. Mohebbi, M. A. Farajzadeh, and M. R. Afshar Mogaddam, "Simultaneous derivatization and air-assisted liquid-liquid microextraction based on solidification of lighter than water deep eutectic solvent followed by gas chromatography-mass spectrometry: an efficient and rapid method for trace analysis of aromatic amines in aqueous samples," Analytica Chimica Acta, vol. 1032, pp. 48-55, 2018.

[20] M. A. Farajzadeh, M. Abbaspour, M. R. A. Mogaddam, and H. Ghorbanpour, "Determination of some synthetic phenolic antioxidants and bisphenol $\mathrm{A}$ in honey using dispersive liquid-liquid microextraction followed by gas chromatographyflame ionization detection," Food Analytical Methods, vol. 8, no. 8, pp. 2035-2043, 2015.

[21] A. Mohebbi, M. A. Farajzadeh, S. Yaripour, and M. R. A. Mogaddam, "Detarmination of tricyclic antidepressants in human urine samples by the three-step sample pretreatment followed by HPLC-UV analysis: an efficient analytical method for further pharmacokinetic and forensic studied," Excel Journal, vol. 17, pp. 952-963, 2018.

[22] Y. Y. Han, N. Deng, J. J. Xie, and Ch. L. Liu, "Acid-assisted dispersive liquid-liquid microextraction-high performance liquid chromatography-tandem mass spectrometry for determination of mycotoxins in fruit juice," Chinese Journal of Analytical Chemistry, vol. 47, pp. 455-462, 2019.

[23] Y. P. Liu, Applications of QuEChERS Method in Detection of Veterinary Drug Residues and Mycotoxins in Complex Matrices, Zhejiang university of technology, Hangzhou, China, 2017.

[24] N. Arroyo-Manzanares, K. De Ruyck, V. Uka et al., "In-house validation of a rapid and efficient procedure for simultaneous determination of ergot alkaloids and other mycotoxins in wheat and maize," Analytical and Bioanalytical Chemistry, vol. 410, no. 22, pp. 5567-5581, 2018.

[25] Sh. M. Wang, Ch. X. Du, X. X. Liu et al., "Simultaneous determination of 26 mycotoxins in Noginseng radix et rhizoma by QuEChERS ultra high performance liquid chromatography tandem mass spectrometry," World Chinese Medicine, vol. 14, no. 4, pp. 798-804, 2019.

[26] H. ., L. Wang, L. Yu, P. W. Li, M. Li, Q. Zhang, and W. Zhang, "Determination of aflatoxin $\mathrm{B}_{1}$ and aflatoxin $\mathrm{B}_{2}$ in edible oil by using graphene oxide- $\mathrm{SiO}_{2}$ as soild phase extraction coupled with HPLC," Chinese Journal Analytical Chemistry, vol. 42, no. 9, pp. 1338-1342, 2014.

[27] G. J. Ma, J. Zhu, L. H. Tang, and Y. Zhang, "Determination of 14 Mycotoxins in lycium seed oil by dispersive solid-phase extraction combined with ultra high performance liquid chromatography-tandem mass spectrometry (UPLC-MS/ MS," Food Fermentation Sciences \& Technology, vol. 55, no. 1, pp. 95-100, 2019.

[28] S. J. Lehotay, M. O’Neil, J. Tully et al., "Determination of pesticide residues in foods by acetonitrile extraction and partitioning with magnesium sulfate: collaborative study," Journal of AOAC International, vol. 90, no. 2, pp. 485-520, 2007.

[29] C. Lesueur, P. Knittl, M. Gartner, A. Mentler, and M. Fuerhacker, "Analysis of 140 pesticides from conventional farming foodstuff samples after extraction with the modified QuEChERS method," Food Control, vol. 19, no. 9, pp. 906914, 2008. 